\title{
Traumatic Brain Injury - Management Principles of Raised Intracranial Pressure and the Emergence of Hypertonic Saline
}

\section{Li Y, Parmar V, Dempsey R, Medow J* \\ Department of Neurological Surgery, University of Wisconsin Medical School, K4/834 CSC, 600 Highland Avenue, Madison, Wisconsin 53792-0001, USA}

*Corresponding author: Medow J, Department of Neurological Surgery, University of Wisconsin Medical School, K4/834 CSC, 600 Highland Avenue, Madison, Wisconsin 53792-0001, USA, Tel: (608) 263-9651; Fax: (608) 263-1728; E-mail: medow@neurosurgery.wisc.edu

Received date: November 23, 2015; Accepted date: December 2, 2015; Published date: December 9, 2015

Copyright: @2015 Medow J et al. This is an open-access article distributed under the terms of the Creative Commons Attribution License, which permits unrestricted use, distribution, and reproduction in any medium, provided the original author and source are credited.

Keywords: Traumatic brain injury; Cerebral edema; Hypertonic saline; Mannitol; Urea; intracranial pressure; ICP

\section{Introduction}

Traumatic brain injury (TBI) is one of the leading causes of premature death and disability in the modern world [1]. Most TBIs occur after motor vehicle accidents and are especially prevalent in the developing world as motorized vehicles are on the rise [2]. In Europe the estimated incidence of TBI is over 200 cases per 100,000 persons per year; therefore, TBI has become a critical public health and socioeconomic problem primarily afflicting younger persons $[3,4]$.

TBI can be classified as primary or secondary brain injury. Primary injury refers to the initial damage caused by the direct impact during a penetrating, blast, or blunt force whereas secondary injury is a consequence of a primary injury whereby local and systemic factors at the cellular level compounds the initial damage. Mediators such as glutamate, arachidonic acid, and leukotrienes have been detected after TBI and are implicated in facilitating secondary injury $[5,6]$.

Secondary brain injury typically develops over the ensuing 5 days following a primary injury and is exacerbated by physiologic perturbations such as hypoxia, hypotension, raised intracranial pressure, and reduced cerebral perfusion pressure all of which aggravate cerebral ischemia and edema. Cerebral edema is an abnormal accumulation of fluid within the brain parenchyma either within the interstitial space (vasogenic) or within cells (cytotoxic) both forms can be seen after TBI.

Vasogenic edema develops as a result of disruption of the blood brain barrier and is commonly seen 24-48 hours after primary injury in the surrounding white matter [7]. Groger et al. hypothesized that disruption of the blood brain barrier associated with the breakdown of extracellular proteins seen after TBI increases the osmotic gradient resulting in water diffusion into the extracellular space thereby potentiating vasogenic edema [8]. The increased osmotic gradient decelerates fluid clearance and facilitates further accumulation of breakdown products resulting in a cyclic potentiation of secondary injury [9].

Cytotoxic edema is the predominant form of edema seen after TBI and develops from cerebral ischemia after the primary injury. Cellular injury initiates a molecular cascade of cell membrane disruption, loss of $\mathrm{Na} / \mathrm{K}$ ATPase, formation of free radicles, and activation of proteases which further disrupt the cell membrane resulting in cell death. The shifts in sodium and potassium between the intracellular and extracellular compartments are directly affected by the loss of $\mathrm{Na} / \mathrm{K}$ ATPase and resultant energy failure. With cell death there is additional accumulation of metabolic break down products (intermediate/ idiogenic osmoles) from the pathologic catabolism of deoxyribonucleic acid within cells which also accounts for a hyperosmotic gradient formed during secondary brain injury. The formation of intermediate osmoles has been demonstrated in experimental models to facilitate cerebral edema and increased ICP after TBI [10].

The treatment of TBI is far more complex given the myriad of governing factors affecting cell death, cerebral edema, and cerebral ischemia. The relative processes that govern these mechanisms are not completely elucidated therefore the most contemporary treatments of TBI focus on avoidance of cerebral ischemia as the focal point of preventing secondary brain injury. These principles form the basis for monitoring and treating cerebral ischemia and raised intracranial pressure [11]. Cerebral ischemia develops when there is a mismatch between cerebral blood flow (CBF) and the metabolic demands of the brain. The brain lacks any significant oxygen reserve therefore even brief periods of decreased CBF can result in ischemic injury. Cerebral autoregulation is one of the hemostatic mechanisms by which the brain prevents cerebral ischemia.

Cerebral autoregulation serves to maintain a steady CBF over a wide range of cerebral perfusion pressures (CPP) (50 to $150 \mathrm{~mm} \mathrm{Hg}$ under normal circumstances) through arteriolar vasodilation or vasoconstriction. This mechanism ensures a constant inflow of oxygen and other nutrients to be delivered to the brain. In severe TBI cerebral autoregulation will be disrupted in an estimated $49-87 \%$ of patients thereby precipitating aberrant CPP and subsequent cerebral ischemia and edema [12-14]. Low CPP increases the morbidity and mortality after TBI [15].

CPP is the summation of an inflow of CBF provided by the mean arterial pressure (MAP) and an outflow produced by intracranial pressure (ICP). In TBI where cerebral autoregulation is impaired, CPP becomes an important estimate (a near linear relationship) of CBF which must be monitored and maintained after severe TBI $[12,13]$. Based on brain tissue oxygen studies, a maintenance CPP threshold of $60-70 \mathrm{~mm} \mathrm{Hg}$ has been suggested to avoid cerebral ischemia [16,17]; higher thresholds are avoided to prevent hyperemia or secondary hemorrhages [18].

Primary therapies aimed toward preventing secondary brain injury are based on the calculations of CPP; therefore, monitoring systemic blood pressure and ICP (via an arterial line and ICP monitor) is necessary. The most recent TBI Guidelines published in 2007 recommend ICP monitoring in all salvageable patients with TBI either documented via head CT or in a patient with a normal head CT but presenting with two or more risk factors including age $>40$ years, systemic hypotension of systolic blood pressure $<90 \mathrm{mmHg}$ systolic, and/or motor posturing [19]. These guidelines also recommend an ICP 
maintenance threshold of $<20 \mathrm{~mm} \mathrm{Hg}$ as a general treatment goal, a change from previous guideline recommendations [19].

The simplest way to reduce ICP is by patient positioning. This includes elevating the head of the bed and avoiding jugular venous compression. On the other end of the spectrum, an indication for surgical management of elevated ICP involves decompressive craniectomy; however, this is in general reserved for medically refractory cases. A randomized controlled trial published in 2011 concluded that decompressive craniectomy reduced ICP and the length of intensive care stay but was associated with higher morbidity and adverse effects compared to best medical therapy [20]. Best medical therapy aims to prevent secondary brain injury by avoiding precipitating factors such as hypoxia and hypotension while optimizing ICP control via patient positioning, sedation, and use of hyperosmolar therapy.

In the modern era, hyperosmolar therapy is very effective and is frequently used as a temporizing measure for ICP control; however, this has not always been the case. During the 1920's Harvey Cushing and his collaborators -Foley and Putnam- thought to repeat the experiments of Weed and McKibben who showed that infusing hypertonic solutions of sodium sulfate or sodium bicarbonate led to reductions of ICP in cats [21]. Although the experiments by Foley and Putman were successful and lead to a rise in the use of hypertonic solutions for the treatment of TBI, the enthusiasm was quickly tempered by reports of respiratory, cardiac, and renal failure in addition to seizures and increased mortality rates [22-23]. In fact by the 1930s, when asked about hypertonic solutions Walter Dandy spoke: "I feel quite confident that no patient has been saved by [this] method, and certainly many have been lost who might otherwise have survived" [24].

It wasn't until 1958 when Dr. Manuchur Javid (the founding chairman of our department at the University of Wiconsin) and Paul Settlage published their seminal paper on the effects of urea in the management of ICP that the use of hypertonic solutions caught on at a national stage [25]. Urea was administered at $100 \mathrm{mg} / \mathrm{kg}$ but could be given up to $1 \mathrm{~g} / \mathrm{kg}$. The mechanism of urea for reducing ICP is a consequence of its diuretic effect which results in an osmotic gradient forcing the diffusion of water out of cerebrovascular tissues [25]. All hypertonic solutions work in a similar fashion acting to decrease ICP through vasoconstriction, reducing cerebral intracellular volume, and erythrocyte cell volume [26,27]. Urea was used by Dr. Javid in over 3200 patients during his career but eventually fell out of favor with the rising popularity of mannitol which was easier to store and prepare, and had a lower side effect profile compared to urea [28]. Today, the most commonly used hyperosmolar agents in the United States include mannitol and hypertonic saline.

Mannitol was first described as an alternative to urea in 1961 by Wise and Chater who advocated for the drug as an alternative therapy which is equally effective but a longer lasting therapy for the treatment of increased ICP [29]. Although urea has a smaller molecular structure compared to mannitol and is theoretically superior as a diuretic mannitol due to its larger size is not readily diffused into tissues and therefore has a greater therapeutic effect [30]. As a result of reduced subcutaneous extravasation, mannitol also has a lower incidence of venous irritation compared to urea [31]. Mannitol is also more easily prepared and stored in solution, can be safely used in patients with renal disease, and has a lower incidence of rebound increases in ICP [31-32]. Later studies also showed that despite its diuretic effects, mannitol can be given in hypotensive patients because it results in an initial intravascular volume expansion that is later followed by eventual diuresis [33]. For these reasons mannitol has become the initial therapy of choice in many centers across the United States for treatment of hypotensive head-injured patients in the trauma bay. Mannitol can be administered as an initial bolus dose of $1 \mathrm{~g} / \mathrm{kg}$ and redosed every six hours as needed with $0.25 \mathrm{~g} / \mathrm{kg}$. The maximal effect of mannitol is seen 15 minutes after initiation of therapy and lasts up to 6 hours. Bolus dosing of mannitol is more effect than a continuous infusions [33]. Caution should be taken in patients with congestive heart failure because of the volume expansion effect and serum sodium and osmolality should be measured and kept below 155 and 320 respectively to avoid developing acute tubular necrosis [33].

Hyperosmolar therapies such as mannitol and urea do not cross the blood brain barrier and therefore exude their therapeutic effects by elevating the serum osmolality; fluid is therefore allowed to shift intravascularly via diffusion and bulk flow thereby reducing vasogenic edema. Diffusion across an osmotic gradient requires an intact blood brain barrier therefore mannitol may be less effective locally surrounding areas of injury; however, it does provide a global effect of decreasing intracranial volume and ICP. Hemodilutional effects of mannitol may also result in increased cerebral perfusion consequently preventing the development of cerebral ischemia.

Hypertonic saline (HS) solutions on the other hand can be given up to serum sodium of 160 and osmolality of 360 before the risk of developing acute tubular necrosis becomes significant. Although initially investigated even prior to the discovery of urea, the research and clinical application of HS was suppressed until its revival by the popularity of urea in the 1950s.

HS can be prepared in various concentrations such as $1.8 \%, 3 \%$, $7.5 \%, 10 \%$, and $23.4 \%$. Concentrations of $3 \%$ or greater require a central line to prevent intravenous (IV) thrombophlebitis of peripheral veins. However, one must note that when the benefits outweigh the risks, peripheral IV administration of 3\% can be used sparingly.

Like mannitol, HS creates an osmotic gradient between the serum and intracranial cells to increase solute transport and reduced ICP; however, HS is not a diuretic. As such it does not reduce intravascular volume and is ideally used in hypotensive patients or patients where hypervolemia is the goal (i.e. SAH patients with vasospasm). Even in terms of affecting renal function mannitol and HS differ. The discrepancy lies in the fact that hypervolemia therapy lowers renal metabolic demand with increased renal blood flow while diuresis has higher renal metabolic demand with reduced renal blood flow. Also, unlike mannitol or other colloid solutions, sodium is dialyzable by continuous veno-venous hemofiltration which is important if adverse effects occur.

The cerebral effects and mechanisms of HS also differ from mannitol and urea. During secondary injury there is a loss of cell membrane ATPase activity resulting in a disruption of ionic homeostasis. The resultant effect is an increase in extracellular $\mathrm{K}$ with a decrease in extracellular $\mathrm{Na}, \mathrm{Ca}$ and $\mathrm{Cl}$. Sustained ionic disturbances facilitate cytotoxic edema by an electrochemical gradient. HS elevates the plasma sodium concentrations which will effectively dehydrate cytotoxic cells by a reverse effect $[34,35]$.

There are a number of complications that can arise from using hypertonic solutions and therefore close monitoring is important. Frequent measurement of electrolytes and osmolarity is central for avoiding iatrogenic salt and volume overload. Hypercholeremic nonanion gap metabolic acidosis can be the consequence of excessive 
intravascular sodium. To reverse the effects of hypernatremia, enteral free water may be helpful despite there being no true free water deficit in these patients rather an elevation of total body sodium. Central pontine myelinolysis $(\mathrm{CPM})$ is another potential iatrogenic effect seen with the administration of hypertonic solutions. Although to this day there have been no reported cases of CPM associated with HS used to treat elevated ICPs in normonatremic patients; in general, sodium should not vary by more than $12 \mathrm{mmol} / \mathrm{L}$ per each 24 hour period. Risk factors for CPM include patients with thiamine deficiency and chronic hyponatremia. Additional adverse effects of HS include cardiac failure, bleeding diathesis, IV phlebitis, and acute renal failure. Although HS is perceived to increase hematocrit and MAP and can be used in multi trauma patients, the use of $1.8 \%$ saline for volume repletion in burn patients resulted in a $400 \%$ increase in the incidence of renal failure and $200 \%$ increase in mortality rates compared to the use of lactated ringer's solution [36].

The advantages of HS include improved gas exchange and immunomodulation, as well as increased cardiac output and MAP. Tseng et al. reported on a series of 10 subarachnoid hemorrhage patients presenting with a Hunt and Hess grades of $>3$ where statistically significant improvements in BP, CPP, ICP, and cerebrovascular flow velocity were seen after bolus administrations of 23.5\% HS [37]. Murphy et al. also demonstrated through a randomized study involving 30 patients with hepatic encephalopathy and secondarily elevated ICP that significant decreases in ICP and vasopressors use can be seen with the administration of HS [38].

A number of subsequent studies have been completed comparing the effects of mannitol and hypertonic saline for the management of ICP. Battison et al. matched $20 \%$ mannitol and $7.5 \%$ hypertonic saline by prospectively treating a series of 9 patients where the authors found mannitol was able to reduce ICP by an average of $7.5 \mathrm{mmHg}$ compared to $13 \mathrm{mmHg}$ with $7.5 \% \mathrm{HS}$. In their study, $7.5 \% \mathrm{HS}$ administration also resulted in a longer durational effect compared to mannitol [39]. A separate prospective randomized control trial comparing $20 \%$ mannitol and 7.5\% HSD in 20 consecutive medically managed patients presenting with severe TBI found persistent elevations of ICP in 10\% of patients treated with HS compared to $70 \%$ with mannitol [40]. The average rate and duration of elevated ICP for HS was 1.9 and 67 minutes compared to 13.3 and 131 minutes for mannitol. Kerwin et al also resonated the superiority of HS compared to mannitol for reducing ICP by reporting on 22 patients with severe TBI receiving either mannitol or $23.4 \%$ HS where the mean ICP reducing within the first hour was $6.4 \mathrm{mmHg}$ for mannitol and $9.3 \mathrm{mmHg}$ for $23.4 \% \mathrm{HS}$ [41]. Patients in this series were more likely to suffer from intractable ICPs when treated mannitol than with HS (26\% versus $8 \%)$. There was however, no statistically significant difference in the duration of ICP control between these two agents. Lastly, through a series of 10 patients, Horn et al. suggest $7.5 \%$ sodium solution to be effective for treating ICP even when other methods of ICP management such as mannitol and pentobarbital coma have failed [42]. On average, 7.5\% HS was able to reduce ICP from $33 \mathrm{mmHg}$ to $19 \mathrm{mmHg}$ within the first hour of bolus administration even when other therapies have been exhausted.

The literature comparing mannitol to HS for the treatment of elevated ICP is based on small series and varying concentrations of HS; therefore, no definitive statements can be made to support the superiority of one therapy over another. What is apparent is the efficacy of hyperosmolar therapy in the treatment of elevated ICP after TBI and the desperate need for a large randomized prospective trial to investigate the optimal treatment strategies for controlling elevated ICP.

\section{References}

1. Pierce CA, Hanks RA (2006) Life satisfaction after traumatic brain injury and the World Health Organization model of disability. Am J Phys Med Rehabil 85: 889-898.

2. Roberts I (1995) China takes to the roads. BMJ 310: 1311-1313.

3. Tagliaferri F, Compagnone C, Korsic M, Servadei F, Kraus J (2006) A systematic review of brain injury epidemiology in Europe. Acta Neurochir (Wien) 148: 255-268.

4. White BC, Krause GS (1993) Brain injury and repair mechanisms: the potential for pharmacologic therapy in closed-head trauma. Ann Emerg Med 22: 970-979.

5. Kempski O, von Andrian U, Schürer L, Baethmann A (1990) Intravenous glutamate enhances edema formation after a freezing lesion. Adv Neurol 52: 219-223.

6. Kimelberg HK, Barron KD, Bourke RS, Nelson LR, Cragoe EJ (1990) Brain anti-cytoxic edema agents. Prog Clin Biol Res 361: 363-385.

7. Marmarou A, Signoretti S, Fatouros PP, Portella G, Aygok GA, et al. (2006) Predominance of cellular edema in traumatic brain swelling in patients with severe head injuries. J Neurosurg 104: 720-730.

8. Gröger U, Marmarou A (1990) Importance of protein content in the edema fluid for the resolution of brain edema. Adv Neurol 52: 215-218.

9. Ito U, Ohno K, Suganuma Y, Suzuki K, Inaba Y (1980) Effect of steroid on ischemic brain edema. Analysis of cytotoxic and vasogenic edema occurring during ischemia and after restoration of blood flow. Stroke 11: 166-172.

10. Pascual JM, Solivera J, Prieto R, Barrios L, López-Larrubia P, et al. (2007) Time course of early metabolic changes following diffuse traumatic brain injury in rats as detected by (1)H NMR spectroscopy. J Neurotrauma 24: 944-959.

11. Robertson CS, Valadka AB, Hannay HJ, Contant CF, Gopinath SP, et al. (1999) Prevention of secondary ischemic insults after severe head injury. Crit Care Med 27: 2086-2095.

12. Fieschi C, Battistini N, Beduschi A, Boselli L, Rossanda M (1974) Regional cerebral blood flow and intraventricular pressure in acute head injuries. J Neurol Neurosurg Psychiatry 37: 1378-1388.

13. Bouma GJ, Muizelaar JP (1992) Cerebral blood flow, cerebral blood volume, and cerebrovascular reactivity after severe head injury. J Neurotrauma 9 Suppl 1: S333-348.

14. Hlatky R, Valadka AB, Robertson CS (2005) Intracranial pressure response to induced hypertension: role of dynamic pressure autoregulation. Neurosurgery 57: 917-923.

15. Struchen MA, Hannay HJ, Contant CF, et al. (2001) The relation between acute physiological variables and outcome on the Glasgow Outcome Scale and Disability Rating Scale following severe traumatic brain injury. J Neurotrauma 18: 115-125.

16. Kiening KL, Härtl R, Unterberg AW, Schneider GH, Bardt T, et al. (1997) Brain tissue pO2-monitoring in comatose patients: implications for therapy. Neurol Res 19: 233-240.

17. Chan KH, Dearden NM, Miller JD, Andrews PJ, Midgley S (1993) Multimodality monitoring as a guide to treatment of intracranial hypertension after severe brain injury. Neurosurgery 32: 547-552.

18. Simard JM, Bellefleur M (1989) Systemic arterial hypertension in head trauma. Am J Cardiol 63: 32C-35C.

19. Shutter L, Timmons SD, Ullman JS, Videtta W, Wilberger JE, et al. (2007) Guidelines for the management of severe traumatic brain injury. VIII. Intracranial pressure thresholds. J Neurotrauma 24: S55-S58.

20. Cooper DJ, Rosenfeld JV, Murray L, et al. (2011) Decompressive craniectomy in diffuse traumatic brain injury. N Engl J Med 364: 1493 1502.

21. Foley FEB, Putnam TJ (1920) The effect of salt ingestion on cerebro-spinal fluid pressure and brain volume. Am J Physiol 53: 464-476. 
Citation: Yiping L, Vikas P, Robert D, Medow J (2015) Traumatic Brain Injury-Management Principles of Raised Intracranial Pressure and the Emergence of Hypertonic Saline . J Neurol Neurophysiol 6: 331. doi:10.4172/2155-9562.1000331

Page 4 of 4

22. Browder J (1930) Dangers in the use of hypertonic solutions in the treatment of brain injuries. Am J Physiol 8: 1213-1217.

23. Anderson WAD, Bethea WR (1940) Renal lesions following administration of hypertonic solutions of sucrose: report of 6 cases. J Am Med Assoc 114: 1983-1987.

24. Dandy WE (1933) Diagnosis and treatment of injuries of the head. J Am Med Assoc 101: 772-775.

25. Javid M, Settlage P, Monfore T (1957) Urea in the management of increased intracranial pressure. Surg Forum 7: 528-532.

26. Muizelaar JP, Wei EP, Kontos HA, Becker DP (1983) Mannitol causes compensatory cerebral vasoconstriction and vasodilation in response to blood viscosity changes. J Neurosurg 59: 822-828.

27. Winn HR, Youmans JR (2004) Youmans Neurological Surgery. (5stedn), W.B. Saunders company, Philadelphia, London, Toranto.

28. Meyer GA (1982) Manucher J. Javid, M.D. Surg Neurol 18: 227-229.

29. Wise BL, Chater N (1961) Effect of mannitol on cerebrospinal fluid pressure. The actions of hypertonic mannitol solutions and of urea compared. Arch Neurol 4: 200-202.

30. Wise BL, Chater N (1961) Use of hypertonic mannitol solutions to lower cerebrospinal fluid pressure and decrease brain bulk in man. Surg Forum 12: 398-399.

31. Bhardwaj A (2007) Osmotherapy in neurocritical care. Curr Neurol Neurosci Rep 7: 513-521.

32. Matson dd (1965) Treatment of cerebral swelling. N Engl J Med 272: 626-628.

33. Bratton SL, Chestnut RM, Ghajar J, et al. (2007) Guidelines for the management of severe traumatic brain injury. II. Hyperosmolar therapy. J Neurotrauma 24: S14-S20.

34. Betz AL (1986) Transport of ions across the blood-brain barrier. Fed Proc 45: 2050-2054.
35. Yan Y, Dempsey RJ, Flemmer A, Forbush B, Sun D (2003) Inhibition of $\mathrm{Na}(+)-\mathrm{K}(+)-\mathrm{Cl}(-)$ cotransporter during focal cerebral ischemia decreases edema and neuronal damage. Brain Res 961: 22-31.

36. Huang PP, Stucky FS, Dimick AR, Treat RC, Bessey PQ, et al. (1995) Hypertonic sodium resuscitation is associated with renal failure and death. Ann Surg 221: 543-554.

37. Tseng MY, Al-Rawi PG, Pickard JD, Rasulo FA, Kirkpatrick PJ (2003) Effect of hypertonic saline on cerebral blood flow in poor-grade patients with subarachnoid hemorrhage. Stroke 34: 1389-1396.

38. Murphy N, Auzinger G, Bernel W, Wendon J (2004) The effect of hypertonic sodium chloride on intracranial pressure in patients with acute liver failure. Hepatology 39: 464-470.

39. Battison C, Andrews PJ, Graham C, Petty T (2005) Randomized, controlled trial on the effect of a $20 \%$ mannitol solution and a $7.5 \%$ saline/ $6 \%$ dextran solution on increased intracranial pressure after brain injury. Crit Care Med 33: 196-202.

40. Vialet Renaud, Albanèse Jacques, Thomachot Laurent, Antonini François, Bourgouin Aurélie, et al. (2003) Isovolume hypertonic solutes (sodium chloride or mannitol) in the treatment of refractory posttraumatic intracranial hypertension: $2 \mathrm{~mL} / \mathrm{kg} 7.5 \%$ saline is more effective than 2 $\mathrm{mL} / \mathrm{kg}$ 20\% mannitol. Crit Care Med 31: 1683-1687.

41. Kerwin AJ, Schinco MA, Tepas JJ, et al. (2009) The use of $23.4 \%$ hypertonic saline for the management of elevated intracranial pressure in patients with severe traumatic brain injury: a pilot study. J Trauma 67: 277-282.

42. Horn P, Münch E, Vajkoczy P, Herrmann P, Quintel M, et al. (1999) Hypertonic saline solution for control of elevated intracranial pressure in patients with exhausted response to mannitol and barbiturates. Neurol Res 21: $758-764$. 